\title{
IN SILICO MEDICINE APPROACH TO PREDICT CHANGES IN HUMAN VERTEBRAE DUE TO OSTEOPOROSIS AND TREATMENT
}

\author{
Levchuk $\mathrm{A}^{1}$, Badilatti $\mathrm{SD}^{1}$, Webster $\mathrm{DJ}^{1}$, van Rietbergen $\mathrm{B}^{2}$, Hazrati Marangalou $\mathrm{J}^{2}$, Ito $\mathrm{K}^{2}$, Müller $\mathrm{R}^{1}$ \\ ${ }^{1}$ Institute for Biomechanics, ETH Zurich, Zurich, Switzerland \\ ${ }^{2}$ Department of Biomedical Engineering, Eindhoven University of Technology, Eindhoven, The Netherlands
}

ALevchuk@ethz.ch

\begin{abstract}
It is generally accepted that trabecular architecture plays a pivotal role in the mechanical behaviour of bone. With age, bone undergoes structural changes, which can result in osteoporosis, leading to lifethreatening fractures, and inevitable decrease in the quality of life. While mathematical laws governing bone remodelling are under continued investigation, the aim of this project was to apply a simple in silico model to simulate changes in the bone architecture due to age, as previously reported in clinical studies. In addition, the effects of the current recommended treatments were investigated. Using high-resolution three-dimensional $\mu C T$ scans of whole human vertebrae, age-related bone loss and recovery simulation produced realistic simulations of structural change over 30 years.
\end{abstract}

Keywords: Bone microstructure, simulation, osteoporosis, pharmaceutical treatment, in silico medicine

\section{Introduction}

Mechanical behaviour of bone depends on the internal trabecular structure and its ability to withstand applied loads. Resistance to stress often becomes impaired due to age-related osteopenia. If left untreated the condition can develop into osteoporosis and lead to potentially lifethreatening bone fractures and reduced quality of life. Current clinical practices lead to accurate diagnosis of reduced bone quality, however cannot always identify individuals that are prone to the development of fractures. Patient-specific analysis of bone architecture and longterm prediction of bone adaptation could lead to improved treatment planning and fracture prevention.

Extensive efforts have been made to develop a deeper insight into the biological phenomena involved in bone remodelling, as well as to translate those processes into mathematical terms. In silico medicine attempts to apply this knowledge to artificially reproduce and predict in vivo events in patients using modelling simulation and visualization techniques of biological and medical processes in computers.

The simulated bone atrophy (SIBA) algorithm [1] was initially developed for the prediction of trabecular bone remodelling due to age-related bone loss. Later, it was successfully applied to model anabolic changes in mouse bone due to mechanical loading [2]. The aim of this project was to extend the application to simulate the effects of osteoporosis, anabolic and antiresorptive treatments, based on the reports of clinical data.

\section{Methods}

SIBA was applied to high-resolution images of 20 whole human vertebral bones (twelfth thoracic vertebra, T12) samples. Images were collected with micro computed tomography $(\mu \mathrm{CT})$ at an isotropic nominal resolution of 37 $\mu \mathrm{m}$. To normalize noise, samples were treated with Gaussian filtration (sigma $=0.6$, support $=1$ ) followed by thresholding prior to the simulations.

SIBA itself also comprised a combination of Gaussian filtration and thresholding. Tab. 1 lists simulation assumptions and selected parameter settings for each scenario. In all cases, activation frequencies and iteration lengths were derived from clinical reports, while model parameters were matched for each condition.

Within the study, simulations of osteoporosis (30 years), antiresorptive (bisphosphonate, BIS) treatment (11 years), and anabolic (parathyroid hormone, PTH) treatment (11 years) have been performed. Two treatment scenarios were considered to mimic realistic clinical settings: in the preventive treatment case, pharmaceutical intervention was started instantaneously; in the late treatment case, simulation of treatment began after 9 years of bone loss. The first year of pharmaceutical treatment was simulated separately for both treatment options, due to the initial increase of up to $6 \% /$ year in bone volume fraction (BV/TV) shown clinically. In the following two years the effect was allowed to plateau in accordance with the clinical reports [2-4]. Despite the fact that treatment with the PTH is currently approved for a maximum of 18 months, simulations were projected to reflect 10 years of treatment for both BIS and PTH cases to allow comparison of microstructural changes due to different simulation scenarios. Simulation outcomes were assessed both morphometrically and visually.

\begin{tabular}{l|cccc}
\hline \multicolumn{1}{c|}{ Scenario } & Sigma & Support & Threshold & $\begin{array}{c}\text { Iteration } \\
\text { (years) }\end{array}$ \\
\hline $\begin{array}{l}\text { Osteoporosis } \\
1^{\text {st }} \text { year }\end{array}$ & 1.2 & 2 & 0.5 & 3 \\
treatment & 1.5 & 3 & 0.4 & 1 \\
BIS & 1.5 & 3 & 0.4 & 10 \\
PTH & 0.7 & 2 & 0.4 & 2.2 \\
\hline Table 1: Summary of & SIBA parameters used for the
\end{tabular}

simulation of osteoporosis and treatment with BIS and PTH.

\section{Results}

Following the selection of corresponding algorithm settings for each scenario, long-term effect of bone loss and 
pharmaceutical treatments have been simulated in 20 vertebral samples (Fig. 1 and 2). The average decrease in bone volume fraction (BV/TV) was $37 \%$ following 30 years of osteoporosis-related bone loss. The first years of treatment, either BIS of PTH related was simulated with the identical settings due to the similarity of the values reported in literature, and resulted in average increases of $5.5 \%$ in $\mathrm{BV} / \mathrm{TV}$. The variance between preventive and late treatment scenarios was $2 \%$. Total increase of $10-12 \%$ in bone volume fraction was simulated in response to BIS treatment for the period of additional 10 years regardless of the start of treatment. In the PTH treatment case, an average increase of $8-11 \%$ in BV/TV was measured at the end of the 10 year simulation.

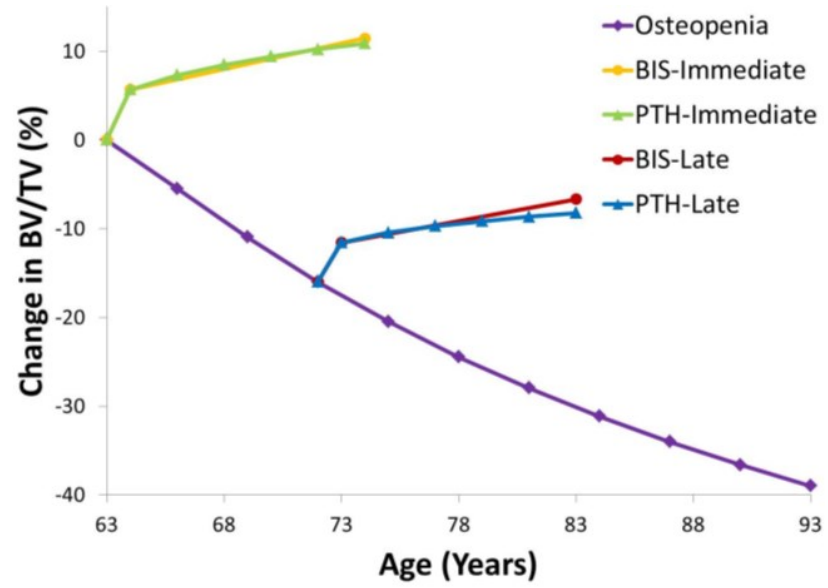

Figure 1: Simulated changes in bone volume fraction (BV/TV) due to osteoporosis and pharmaceutical treatments in a 63 year-old female.

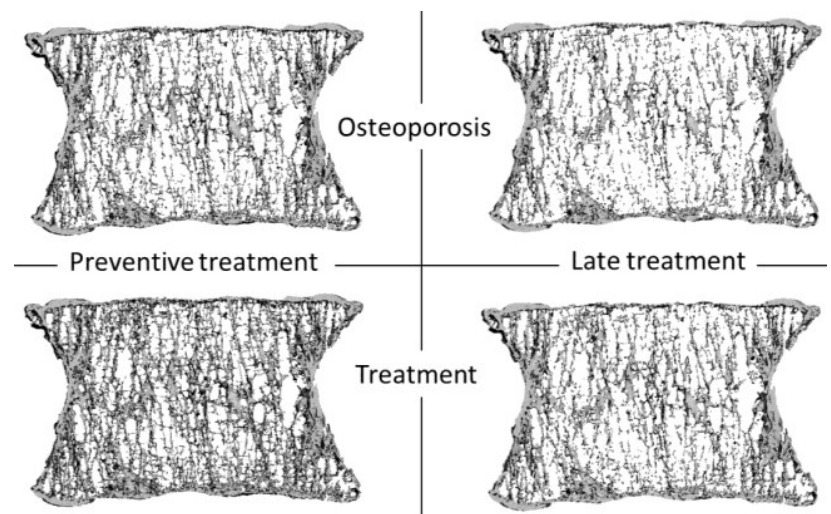

Figure 2: In silico modelling of microstructural changes in human bone due to osteoporosis and pharmaceutical treatments. Left: Effect of the preventive treatment scenario (74-75 years of age). Right: Corresponding results for the late treatment scenario (83-84 years of age). Top: Bone structure affected by osteoporosis. Bottom: Corresponding structural changes following pharmaceutical treatment.

\section{Discussion}

The goal of this project was to simulate microstructural changes in bone architecture due to osteoporosis and related pharmaceutical treatments in whole human bone samples. SIBA has been successfully employed to model long-term effects of both osteopenia and bone formation processes in $3 \mathrm{D}$ high-resolution images. The outcomes of the simulations performed in 20 human vertebrae have both realistic trabecular architecture, and morphometric indexes comparable to clinical findings.

For example, Müller [1] reported an average decrease of $38 \%$ in vertebral BV/TV following 30 years of menopause, a results that could be recreated exactly in the current implementation of the algorithm. Clinical reports of bone changes after the first year of treatment with either PTH [3, 4] or BIS [5], were in the range of 6\% increase across all studies, and could also be accurately reflected in the simulations. Projected in silico result for the BIS treatment matched expected changes in clinics, as reported by Seeman and Delmas [5], despite the challenge of a single 10 yearlong iteration required to match the physiological parameters. Simulated PTH treatment aligned well with the data reported for the 18 months long administration of the drug, in which an average increase of $6.5 \%$ was reported for the lumbar spine measurements [3].

In conclusion, in silico medicine is a powerful tool that not only accurately reflects biological events in bone, but can also predict changes in the microstructure. With the implementation of the models for the whole threedimensional human samples, the effects of bone loss, as well as bone formation could be realistically simulated. Linking current computational capabilities and clinical practice could not only improve the accuracy of the diagnosis, but also contribute to patient-specific treatment planning.

\section{Acknowledgement}

Funding from the EU for the osteoporotic virtual physiological human project (VPHOP FP7-ICT2008$223865)$ is gratefully acknowledged.

\section{Bibliography}

[1] R. Müller, "Long-term prediction of three-dimensional bone architecture in simulations of pre-, peri- and postmenopausal microstructural bone remodeling," Osteoporosis International, vol. 16, pp. S25-S35, Mar 2005.

[2] F. A. Gerhard, D. J. Webster, G. H. van Lenthe, and R. Müller, "In silico biology of bone modelling and remodelling: adaptation," Philosophical transactions. Series A, Mathematical, physical, and engineering sciences, vol. 367, pp. 2011-30, May 282009.

[3] E. Jodar-Gimeno, "Full length parathyroid hormone (184 ) in the treatment of osteoporosis in postmenopausal women," Clinical Interventions in Aging, vol. 2, pp. 163-174, 2007.

[4] J. R. Zanchetta, C. E. Bogado, C. Cisari, S. Aslanidis, H. Greisen, J. Fox, and W. Lems, "Treatment of postmenopausal women with osteoporosis with $\mathrm{PTH}(1-$ 84) for 36 months: treatment extension study," Current Medical Research and Opinion, vol. 26, pp. 26272633, Nov 2010

[5] E. Seeman and P. D. Delmas, "Bone quality--the material and structural basis of bone strength and fragility," The New England journal of medicine, vol. 354, pp. 2250-61, May 252006. 